\title{
Parallel-Distributed Model Deformation in the Fingertips for Stable Grasping and Object Manipulation
}

\author{
R. García-Rodríguez ${ }^{1}$ and G. Díaz-Rodríguez ${ }^{2}$ \\ ${ }^{1}$ Facultad de Ingeniería y Ciencias Aplicadas, Universidad de los Andes, \\ Av. San Carlos de Apoquindo 2200, Las Condes, Santiago, Chile \\ ${ }^{2}$ Departamento de Ingeniería Eléctrica, Universidad de Chile, Av. Tupper 2007, Santiago, Chile \\ Correspondence should be addressed to R. García-Rodríguez, rgarcia1@miuandes.cl
}

Received 27 April 2012; Revised 13 July 2012; Accepted 25 July 2012

Academic Editor: J. Rodellar

Copyright (c) 2012 R. García-Rodríguez and G. Díaz-Rodríguez. This is an open access article distributed under the Creative Commons Attribution License, which permits unrestricted use, distribution, and reproduction in any medium, provided the original work is properly cited.

\begin{abstract}
The study on the human grip has inspired to the robotics over the past decades, which has resulted in performance improvements of robotic hands. However, current robotic hands do not have the enough dexterity to execute complex tasks. Recognizing this fact, the soft fingertips with hemispherical shape and deformation models have renewed attention of roboticists. A high-friction contact to prevent slipping and the rolling contribution between the object and fingers are some characteristics of the soft fingertips which are useful to improve the grasping stability. In this paper, the parallel distributed deformation model is used to present the dynamical model of the soft tip fingers with $n$-degrees of freedom. Based on the joint angular positions of the fingers, a control scheme that fuses a stable grasping and the object manipulation into a unique control signal is proposed. The force-closure conditions are defined to guarantee a stable grasping and the boundedness of the closed-loop signals is proved. Furthermore, the convergence of the contact force to its desired value is guaranteed, without any information about the radius of the fingertip. Simulation results are provided to visualize the stable grasping and the object manipulation, avoiding the gravity effect.
\end{abstract}

\section{Introduction}

From physiological point of view, the human hands are considered as a powerful tool whereby the human brain interacts with the world, that is, how it perceives and acts with the environment [1]. In order to increase the dexterity in robotic hands, some intelligent humanlike functions have been imitated.

In general, the dexterous manipulation in robotics, to emulate pinching motions, have been formulated in terms of the object, that is, the forces/torques exerted on it to produce the desired movements and how they behave [2]. The grasping and the object manipulation are 
based on the assumption that the contact between the object and fingers is frictionless, so the finger can only exert a force along the common normal axis at the contact point [3]. Then, to grasp the object without slipping the standard friction cone is used, generating a complex motion control since their evolution is governed by the laws of Coulomb friction, which is nonlinear and imposes constraints on the system. On the other hand, some authors have considered fingers with a very sharp curvature assuming that the contact point between the fingers and object does not change significantly. Although, some manipulation tasks are executed by robotic fingers successfully, this assumption is not valid for several manipulation tasks because the rolling between object and fingers is essential in the human manipulation tasks. Moreover, some attempts to determine the best grasp configuration and manipulation tasks are presented in 2D and 3D [4-11]. Unfortunately, a lot of them require an exact knowledge of the system parameters and the object localization [12-15]. Some authors, to reproduce more characteristics of the human fingertips, have considered the use of deformation models with hemispherical soft tips. Many hemispherical soft tip fingers have been designed and constructed to execute several manipulation tasks [16-19] where a high-contact friction to prevent slipping and the rolling of the finger tip on the object surface are some characteristics $[3,20,21]$. Nevertheless, in these approaches the contribution of the fingertip deformation on the manipulation tasks in a dynamic sense is not evident, considering that the rolling constraint is defined in kinematic or semidynamics sense [21-24].

On the basis that human hands have fingers with soft tips, in this paper the grasping and the object manipulation is presented, using a pair of robotic fingers with $n$-degree of freedoms and deformable tips. The parallel deformation model is based on a virtual spring with infinitesimal section, where the normal and tangential deformations are taking into account [25]. So, a tangential movement of the object without slipping and a dependency of the relative orientation between the object and the finger are considered. Inclusion of the normal and tangential deformations in the deformation model, contribute to reproduce some intrinsic characteristics of the deformable material and a better grip. The key of our approach is to introduce the parallel deformation model to grasp an object using a pair of robotic fingers with $n$-degrees of freedom. Moreover, the grasping controller guaranteed that the contact force converges to the desired value, avoiding a direct dependence with the radius of the tip [26, 27]. An approximation of the object angle based on the joint angular position of the fingers is used to control orientation of the object. Finally, a control signal for translation of the object is defined. To carry out the grasping and the object manipulation, the superposition principle is used $[28,29]$, which allows us to separate a complex task into a set of basic tasks, where each task has a unique stationary point which represents the desired action [27]. Boundedness of all closed-loop signals is proved, while the asymptotic stability is guaranteed using the stability on the manifold $[27,28,30]$. It is important to notice that forces-closure conditions, to grasp firmly an object, are satisfied dynamically during manipulation task execution, rather than a static equilibrium. The proposed approach is validated by numerical simulations through a pair of robotic fingers with soft tips in the horizontal plane.

This paper is organized as follows. Section 2 presents dynamical equations of the fingers-object system. The blind control law is proposed in Section 3. Simulation results to confirm the validity of our approach are presented in Section 4 . Finally, the conclusions are presented in Section 5.

\section{Dynamical Equations}

Consider a pair of soft tip fingers, with three degree of freedom each one, grasping an object in the horizontal plane, as shown in Figure 1. In the fingers-object system $O$ is the origin for 


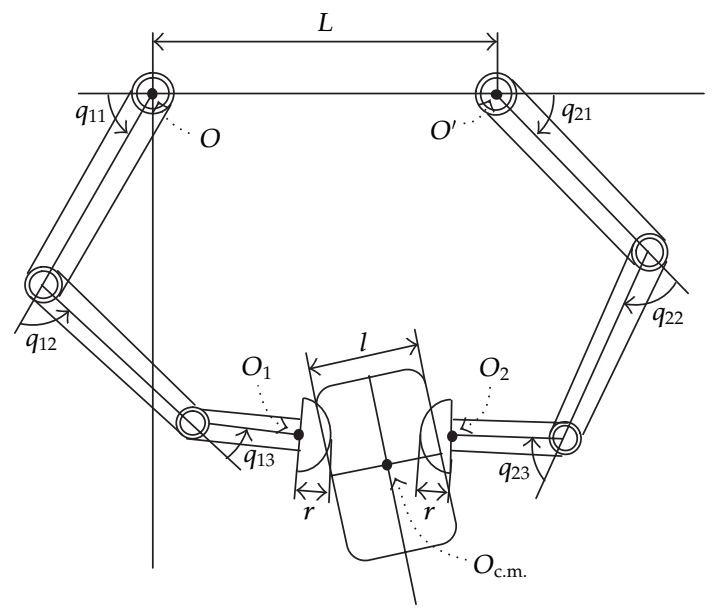

Figure 1: Rigid object grasped by a pair soft tip fingers.

the left finger and it is considered as the reference frame, $O^{\prime}$ is the origin for the right finger, and $L$ is the distance between the origins of each finger. In addition, $q_{i}=\left[q_{i 1}, q_{i 2}, q_{i 3}\right]^{T}$ is the joint angular positions of the finger $i, r_{i}$ is the radius of the soft tip finger $i, l$ is the length of the object, $l_{i j}$ is the length of the link $j$ for the finger $i, O_{i}=\left(x_{i}, y_{i}\right)$ is the center position of the deformable fingertips $i$ with $i=1,2, O_{\text {c.m. }}=(x, y)$ is the center of mass of the object, and $\theta$ is the orientation of the object. Unlike deformation model proposed by $[22,28]$, where the force applied to the object produce a distribute pressure and it is parametrized as a normal force $f_{i}$ with respect to the object surface. In this paper we use the deformation model proposed in [25], where a virtual spring inside the soft tip finger allows us to known the normal and tangential movements in the deformable material. In such a way, the elastic energy induced by the soft fingertip $i$ is given as

$$
P_{i}\left(d_{n i}, d_{t i}, \phi_{i}\right)=\pi E\left[\frac{d_{n i}^{3}}{3 \cos ^{2}\left(\phi_{i}\right)}+d_{n i}^{2} d_{t i} \tan \left(\phi_{i}\right)+d_{n i} d_{t i}^{2}\right],
$$

where $d_{n i}$ is the maximum radial deformation, $\phi_{i}$ is the object relative orientation angle, $d_{t i}$ is the contact tangential displacement of the object, and $E$ is the Young's modulus of the finger tip material, as shown in Figure 2. Thus, the total potential energy of the deformable fingertips is expressed as

$$
P=P_{1}\left(d_{n 1}, d_{t 1}, \phi_{1}\right)+P_{2}\left(d_{n 2}, d_{t 2}, \phi_{2}\right)
$$

The constraint between the radial deformation of the fingertip $i$ and the object is given as

$$
C_{n i}=-\left[\left(r_{i}-d_{n i}\right)+l_{i}+(-1)^{i}\left(\left(x-x_{i}\right) \cos (\theta)-\left(y-y_{i}\right) \sin (\theta)\right)\right]=0,
$$

which guarantees that exists a distance that limits the grasping on the object in normal direction. A particular case of (2.3) is considered when $d_{n i}=0$ which represents the normal 


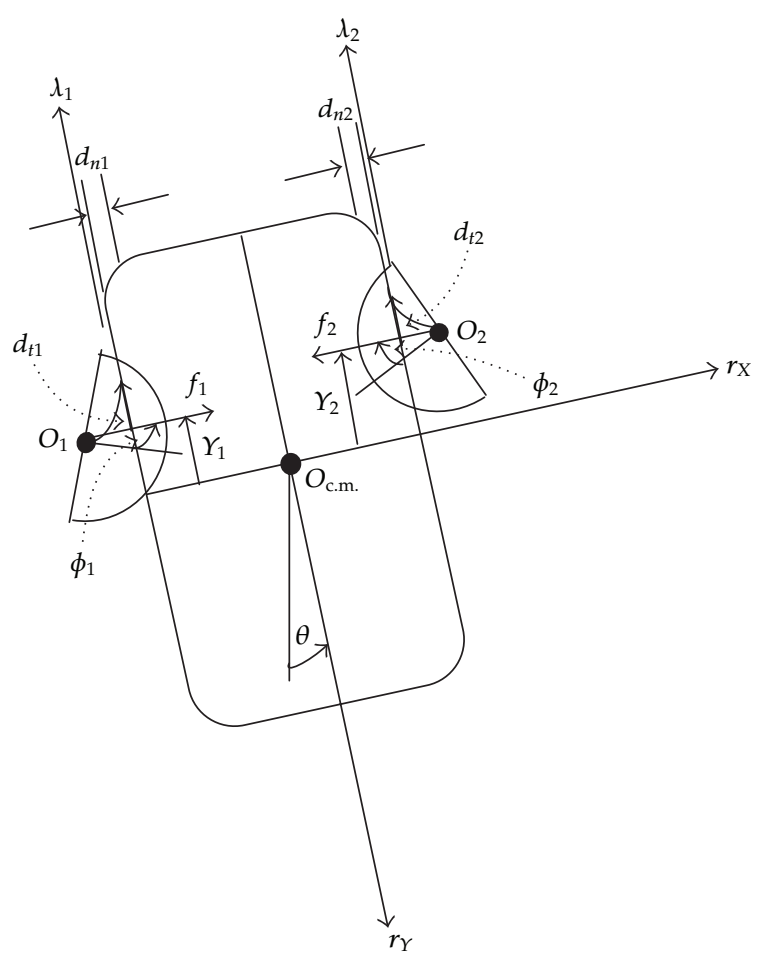

Figure 2: Deformation Model proposed by [25].

constraint for a rigid fingertip as reported in [30]. Accordingly, the normal constrained for the fingers-object system is defined as

$$
C_{n}=\sum_{i} f_{i} C_{n i}
$$

where $f_{i}$ is the Lagrange multiplier and represents the contact force $i$.

On the other hand, taking into account the curvature effects of the fingertip, the rolling of the object on the soft tip finger will be defined as movement of the contact area, where the relative velocity of the contact area between the finger tips and the object is zero. Assuming that the normal deformation $d_{n i}$ is smaller than the radius of the tip $i$ [25], the angular displacement of the contact area and the projection displacement of the center of mass of the object is defined as

$$
\dot{Y}_{i}=-r \dot{\phi}_{i}
$$

where

$$
\begin{gathered}
Y_{i}=\left(x_{i}-x\right) \sin (\theta)+\left(y_{i}-y\right) \cos (\theta), \\
\phi_{i}=\pi-(-1)^{i} \theta-e_{i}^{T} q_{i}
\end{gathered}
$$


and $e_{i}=[1, \ldots, 1]^{T}$ of the same size as the vector $q_{i}$, for $i=1,2$. Moreover, according to the deformation model, a tangential displacement $d_{t i}$ on the deformable fingertip $i$ arises when the object is rolling on the fingertip. Then, the rolling constraint between finger tip $i$ and the object surface is given as

$$
C_{t i}=Y_{i}-c_{S i}+r_{i} \phi_{i}+d_{t i}=0
$$

where $c_{S i}$ is the integration constant with respect to the initial conditions of contact. To avoid initial conditions in (2.7) a velocity constraint is defined as [25]

$$
\dot{C}_{t i}=\dot{Y}_{i}+r_{i} \dot{\phi}_{i}+\dot{d}_{t i}=0
$$

The Lagrangian of the system with holonomic constraint is described by

$$
L=K-P+C_{n}
$$

where $P$ and $C_{n}$ are defined in (2.2) and (2.4), respectively, and $K$ is the kinetic energy of the system defined as

$$
K=\Sigma_{i} \frac{1}{2}\left[\dot{q}_{i}^{T} H_{i}\left(q_{i}\right) \dot{q}_{i}+m_{n i} \dot{d}_{n i}^{2}+m_{t i} \dot{d}_{t i}^{2}\right]+\frac{1}{2} \dot{p}^{T} H_{0} \dot{p},
$$

with $H_{i}\left(q_{i}\right)$ is the inertia matrix of the finger $i, H_{0}=\operatorname{diag}(m, m, I), p=[x, y, \theta]^{T}$, and $m_{n i}$, $m_{t i}$ are the normal and tangential mass deformations, respectively. Applying the Lagrangian variational principle, incorporating the rolling velocity constraint, the equations of motion are expressed for each component of the vector as follows:

$$
\frac{d}{d t}\left(\frac{\partial L}{\partial \dot{z}}\right)-\frac{\partial L}{\partial z}=\frac{\partial}{\partial \dot{z}}\left(\lambda_{1} \dot{C}_{t 1}+\lambda_{2} \dot{C}_{t 2}\right)
$$

where $z=\left[q_{1}^{T}, q_{2}^{T}, x, y, \theta, d_{n 1}, d_{n 2}, d_{t 1}, d_{t 2}\right]^{T}$ is the vector of generalized coordinates and $\lambda_{i}$ is the Lagrange multiplier which represents the tangential force exerted for the finger $i$ on the object surface. Note that treatment of the velocity constraint should not be done in the Lagrangian (2.9), but rather in the equations of motion [31].

Thus, the equations of motion for the fingers are given as

$$
\begin{aligned}
& H_{i}\left(q_{i}\right) \ddot{q}_{i}+\frac{1}{2} \dot{H}_{i}\left(q_{i}\right) \dot{q}_{i}+S_{i}\left(q_{i}, \dot{q}_{i}\right) \dot{q}_{i}-(-1)^{i} f_{i} J_{i}^{T} r_{X}-\lambda_{i}\left(J_{i}^{T} r_{Y}-r e_{i}\right) \\
& -\frac{\pi E d_{n i}^{2}}{\cos ^{2}\left(\phi_{i}\right)}\left(d_{t i}+\frac{2}{3} d_{n i} \tan \left(\phi_{i}\right)\right) e_{i}=u_{i},
\end{aligned}
$$

where $r_{X}=[\cos (\theta), \sin (\theta)]^{T}, r_{Y}=[\sin (\theta), \cos (\theta)]^{T}, J_{i}$ is the Jacobian of the point $\left(x_{i}, y_{i}\right)$ with respect to the joint variables $q_{i j}, e_{i}=[1,1,1]^{T},(1 / 2) \dot{H}_{i}\left(q_{i}\right)+S_{i}\left(q_{i}, \dot{q}_{i}\right)$ represent the matrix of Coriolis and centripetal forces, and $u_{i}$ stands for the torque input. It is important to notice that 
the moments induced by the tangential and normal forces contribute to grasp and manipulate an object more securely. In addition, the terms of deformation model give us information about the behavior of the deformable material. Furthermore, the movement equations of the object are given as

$$
\begin{gathered}
m \ddot{x}-\left(f_{1}-f_{2}\right) \cos (\theta)+\left(\lambda_{1}+\lambda_{2}\right) \sin (\theta)=0, \\
m \ddot{y}+\left(f_{1}-f_{2}\right) \sin (\theta)+\left(\lambda_{1}+\lambda_{2}\right) \cos (\theta)=0, \\
I \ddot{\theta}-\Upsilon_{1} f_{1}+\Upsilon_{2} f_{2}-\lambda_{1}\left(d_{n 1}-l_{1}\right)+\lambda_{2}\left(d_{n 2}-l_{2}\right)+\frac{\pi E d_{n 1}^{2}}{\cos ^{2}\left(\phi_{1}\right)}\left(d_{t 1}+\frac{2}{3} d_{n 1} \tan \left(\phi_{1}\right)\right) \\
-\frac{\pi E d_{n 2}^{2}}{\cos ^{2}\left(\phi_{2}\right)}\left(d_{t 2}+\frac{2}{3} d_{n 2} \tan \left(\phi_{2}\right)\right)=0 .
\end{gathered}
$$

The last two terms of (2.15) represent the contribution of the deformable fingertips to assure a stable grasping through induced forces/moments. Finally, dynamical equations related to the normal $d_{n i}$ and tangential $d_{t i}$ movements on the fingertips are defined as

$$
\begin{gathered}
m_{n i} \ddot{d}_{n i}+\pi E\left(\frac{d_{n i}^{2}}{\cos ^{2}\left(\phi_{i}\right)}+2 d_{n i} d_{t i} \tan \left(\phi_{i}\right)+d_{t i}^{2}\right)-f_{i}=0, \\
m_{t i} \ddot{d}_{t i}+\pi E\left(d_{n i}^{2} \tan \left(\phi_{i}\right)+2 d_{n i} d_{t i}\right)-\lambda_{i}=0 .
\end{gathered}
$$

Summing the products between $\dot{q}_{i}^{T}$ with (2.12), $\dot{x}$ with (2.13), $\dot{y}$ with (2.14), $\dot{\theta}$ with (2.15), $\dot{d}_{n i}$ with (2.16), and $\dot{d}_{t i}$ with (2.17) yields

$$
\sum_{i=1,2} \int_{0}^{t}\left(\dot{q}_{i}^{T} u_{i}\right) d \tau=E(t)-E(0) \geq-E(0),
$$

where $E=K+P$ corresponds to the total energy of the system.

\section{Controller Design}

\subsection{Immobilization on the Object}

As first step before to execute manipulation tasks, a stable grasping must be established. To grasp stably an object, the force-closure is used to guarantee that the object should be held securely by the fingers. This mean that maintaining the contact between the fingers and 
the object, that is, $f_{1}>0$ and $f_{2}>0$ for any $t>0$, the forces and torques applied on the object should immobilize it. Let the forces and torques applied on the object be defined as

$$
\begin{gathered}
-\left(f_{1}-f_{2}\right) \cos (\theta)+\left(\lambda_{1}+\lambda_{2}\right) \sin (\theta)=0, \\
\left(f_{1}-f_{2}\right) \sin (\theta)+\left(\lambda_{1}+\lambda_{2}\right) \cos (\theta)=0, \\
-\Upsilon_{1} f_{1}+\Upsilon_{2} f_{2}+\lambda_{1}\left(d_{n 1}-l_{1}\right)-\lambda_{2}\left(d_{n 2}-l_{2}\right)+\frac{\pi E d_{n 1}^{2}}{\cos ^{2}\left(\phi_{1}\right)}\left(d_{t 1}+\frac{2}{3} d_{n 1} \tan \left(\phi_{1}\right)\right) \\
-\frac{\pi E d_{n 2}^{2}}{\cos ^{2}\left(\phi_{2}\right)}\left(d_{t 2}+\frac{2}{3} d_{n 2} \tan \left(\phi_{2}\right)\right)=0 .
\end{gathered}
$$

Then, if we choose that

$$
f_{1}=f_{2}=f_{d}, \quad \lambda_{1}+\lambda_{2}=0,
$$

the first two equations are equal to zero, while the third equation is given as

$$
\begin{aligned}
& -f_{d}\left(Y_{1}-Y_{2}\right)+\lambda_{1}\left(d_{n 1}-l_{1}+d_{n 2}-l_{2}\right)+\frac{\pi E d_{n 1}^{2}}{\cos ^{2}\left(\phi_{1}\right)}\left(d_{t 1}+\frac{2}{3} d_{n 1} \tan \left(\phi_{1}\right)\right) \\
& -\frac{\pi E d_{n 2}^{2}}{\cos ^{2}\left(\phi_{2}\right)}\left(d_{t 2}+\frac{2}{3} d_{n 2} \tan \left(\phi_{2}\right)\right)=0 .
\end{aligned}
$$

Thus, a force-closure can be established, if the forces acting on the object are defined as,

$$
\begin{gathered}
f_{i} \longrightarrow f_{d}, \quad Y_{1}-Y_{2} \longrightarrow 0, \quad \lambda_{i} \longrightarrow 0, \\
\frac{\pi E d_{n 1}^{2}}{\cos ^{2}\left(\phi_{1}\right)}\left(d_{t 1}+\frac{2}{3} d_{n 1} \tan \left(\phi_{1}\right)\right)-\frac{\pi E d_{n 2}^{2}}{\cos ^{2}\left(\phi_{2}\right)}\left(d_{t 2}+\frac{2}{3} d_{n 2} \tan \left(\phi_{2}\right)\right) \longrightarrow 0 \quad \text { for } i=1,2,
\end{gathered}
$$

where $f_{d}$ is the desired normal force. Once fingers grasp an object and hold it securely, we are in conditions to execute manipulation tasks on the object as orientation and move it at $x-y$ coordinates.

Inspired that humans can execute some manipulation tasks without any object information, in this paper a stable grasping and the object manipulation based only on the center position of the soft tip finger $\left(x_{i}, y_{i}\right)$ are presented, so that the orientation $\theta$ and the object parameters are avoided. Using the superposition principle, the joint torque $u_{i}$ applied to each finger can be defined as

$$
\begin{aligned}
u_{i} & =u_{f c i}+u_{\theta c i} \\
& =-c_{i} \dot{q}_{i}+(-1)^{i} \frac{f_{d}}{\bar{l}} J_{i}^{T}\left(\begin{array}{l}
x_{1}-x_{2} \\
y_{1}-y_{2}
\end{array}\right)+(-1)^{i} \beta \frac{\Delta \overline{\tan (\theta)}}{x_{2}-x_{1}} J_{i}^{T}\left(\begin{array}{c}
\tan (\theta) \\
1
\end{array}\right), \quad i=1,2,
\end{aligned}
$$


where $c_{i}$ is a diagonal symmetric positive definite matrix, $\beta>0, f_{d}>0$ is the desired contact force, $\Delta \overline{\tan (\theta)}=\overline{\tan (\theta)}-\tan \left(\theta_{d}\right), \theta_{d}$ is the desired angle of rotation, and

$$
\begin{gathered}
\overline{\tan (\theta)}=\frac{y_{1}-y_{2}}{x_{2}-x_{1}} \\
\bar{l}^{2}=\left(x_{1}-x_{2}\right)^{2}+\left(y_{1}-y_{2}\right)^{2}=l_{w}^{2}+\left(Y_{1}-Y_{2}\right)^{2} \quad \text { where } l_{w}=r_{1}+r_{2}+l-d_{n 1}-d_{n 2} .
\end{gathered}
$$

Notice that $u_{f c i}$ refers to the stable grasp exerted on the object, while $u_{\theta c i}$ indicates the orientation control of the object. In the former case, a stable grasp is achieved minimizing the distance between the centers of the fingertips through the normal and tangential forces, which guarantee the control objectives $\Delta f_{i} \rightarrow 0, \Delta Y \rightarrow 0$. In the latter case, to avoid the measurement of $\theta$, an approximation of $\theta$ is proposed by the trigonometric tangent function $\overline{\tan (\theta)}$. This approximation has the feature that the convergence of $\overline{\tan (\theta)}$ to $\tan (\theta)$ is guaranteed once $\Delta Y \rightarrow 0$ is satisfied. This implies that the conditions of the stable grasping $\Delta f_{i} \rightarrow$ $0, \Delta Y \rightarrow 0$, and $\overline{\tan (\theta)}$ should be satisfied.

To start the analysis and to ensure a stable grasping, it is considered that the joint torque is defined as $u_{i}=u_{f c i}$. Substituting $u_{i}$ in (2.12), the closed-loop system equations are defined as

$$
\begin{gathered}
H_{i}\left(q_{i}\right) \ddot{q}_{i}+\frac{1}{2} \dot{H}_{i}\left(q_{i}\right) \dot{q}_{i}+S_{i}\left(q_{i}, \dot{q}_{i}\right) \dot{q}_{i}-(-1)^{i} \Delta f_{i} J_{i}^{T} r_{x}-\Delta \lambda_{i}\left(J_{i}^{T} r_{y}-r_{i} e_{i}\right) \\
-\frac{\pi E d_{n i}^{2}}{\cos ^{2}\left(\phi_{i}\right)}\left(d_{t i}+\frac{2}{3} d_{n i} \tan \left(\phi_{i}\right)\right) e_{i}-(-1)^{i} \frac{f_{d}}{\bar{l}} \Delta Y r_{i} e_{i}=-c_{i} \dot{q}_{i} \\
m \ddot{x}-\left(\Delta f_{1}-\Delta f_{2}\right) \cos (\theta)+\left(\Delta \lambda_{1}+\Delta \lambda_{2}\right) \sin (\theta)=0 \\
m \ddot{y}+\left(\Delta f_{1}-\Delta f_{2}\right) \sin (\theta)+\left(\Delta \lambda_{1}+\Delta \lambda_{2}\right) \cos (\theta)=0 \\
I \ddot{\theta}-\Delta f_{1} Y_{1}+\Delta f_{2} \Upsilon_{2}-\Delta \lambda_{1}\left(d_{n 1}-l_{1}\right)+\Delta \lambda_{2}\left(d_{n 2}-l_{2}\right) \\
+\sum_{i=1}^{2}(-1)^{i} \frac{\pi E d_{n i}^{2}}{\cos ^{2}\left(\phi_{i}\right)}\left(d_{t i}+\frac{2}{3} d_{n i} \tan \left(\phi_{i}\right)\right)-\frac{f_{d}}{\bar{l}} \Delta Y\left(r_{1}+r_{2}\right)=0, \\
m_{n i} \ddot{d}_{n i}+\pi E\left(\frac{d_{n i}^{2}}{\cos ^{2}\left(\phi_{i}\right)}+2 d_{n i} d_{t i} \tan \left(\phi_{i}\right)+d_{t i}^{2}\right)-\Delta f_{i}-\frac{l_{w}}{\bar{l}} f_{d}=0, \\
m_{t i} \ddot{d}_{t i}+\pi E\left(d_{n i}^{2} \tan \left(\phi_{i}\right)+2 d_{n i} d_{t i}\right)-\Delta \lambda_{i}+(-1)^{i} \frac{f_{d}}{\bar{l}} \Delta Y=0,
\end{gathered}
$$

where $\Delta f=f_{i}-f_{d} \frac{l_{w}}{\bar{l}}, \Delta Y=Y_{1}-Y_{2}$, and $\Delta \lambda_{i}=\lambda_{i}+(-1)^{i} \frac{f_{d}}{\bar{l}} \Delta Y$. that

Expressing the closed-loop system equations (3.7) in a vector-matrix equation we have

$$
H \ddot{z}+C \dot{z}+P(z)-A \Delta \lambda-D \Delta Y+F \dot{z}=0,
$$


where

$$
\begin{aligned}
& H=\operatorname{diag}\left(H_{1}\left(q_{1}\right), H_{2}\left(q_{2}\right), m, m, I, m_{n 1}, m_{n 2}, m_{t 1}, m_{t 2}\right), \\
& C=\operatorname{diag}\left(\frac{1}{2} \dot{H}_{1}\left(q_{1}\right)+S_{1}\left(q_{1}, \dot{q}_{1}\right), \frac{1}{2} \dot{H}_{2}\left(q_{2}\right)+S_{2}\left(q_{2}, \dot{q}_{2}\right), 0,0,0,0,0,0,0\right), \\
& F=\operatorname{diag}\left(c_{1}, c_{2}, 0,0,0,0,0,0,0\right), \\
& D=\left[-\frac{f_{d}}{\bar{l}} r_{1} e_{1}, \frac{f_{d}}{\bar{l}} r_{2} e_{2}, 0,0, \frac{f_{d}}{\bar{l}}\left(r_{1}+r_{2}\right), \frac{l_{w}}{\bar{l} \Delta Y} f_{d}, \frac{l_{w}}{\bar{l} \Delta Y} f_{d}, \frac{f_{d}}{\bar{l}},-\frac{f_{d}}{\bar{l}}\right]^{T}, \\
& \Delta \mathcal{l}=\left[\Delta f_{1}, \Delta f_{2}, \Delta \lambda_{1}, \Delta \lambda_{2}\right]^{T}, \\
& P(z)=\left[\begin{array}{c}
-\frac{\pi E d_{n 1}^{2}}{\cos ^{2}\left(\phi_{1}\right)}\left(d_{t 1}+\frac{2}{3} d_{n 1} \tan \left(\phi_{1}\right)\right) e_{1} \\
-\frac{\pi E d_{n 2}^{2}}{\cos ^{2}\left(\phi_{2}\right)}\left(d_{t 2}+\frac{2}{3} d_{n 2} \tan \left(\phi_{2}\right)\right) e_{2} \\
0 \\
0 \\
\sum_{i=1}^{2}(-1)^{i} \frac{\pi E d_{n i}^{2}}{\cos ^{2}\left(\phi_{i}\right)}\left(d_{t i}+\frac{2}{3} d_{n i} \tan \left(\phi_{i}\right)\right) \\
\pi E\left(\frac{d_{n 1}^{2}}{\cos ^{2}\left(\phi_{1}\right)}+2 d_{n 1} d_{t 1} \tan \left(\phi_{1}\right)+d_{t 1}^{2}\right) \\
\pi E\left(\frac{d_{n 2}^{2}}{\cos ^{2}\left(\phi_{2}\right)}+2 d_{n 2} d_{t 2} \tan \left(\phi_{2}\right)+d_{t 2}^{2}\right) \\
\pi E\left(d_{n 1}^{2} \tan \left(\phi_{1}\right)+2 d_{n 1} d_{t 1}\right) \\
\pi E\left(d_{n 2}^{2} \tan \left(\phi_{2}\right)+2 d_{n 2} d_{t 2}\right)
\end{array}\right], \\
& A=\left[\begin{array}{cccc}
-J_{1}^{T} r_{X} & 0_{3 \times 1} & J_{S_{q 1}} & 0_{3 \times 1} \\
0_{3 \times 1} & J_{2}^{T} r_{X} & 0_{3 \times 1} & J_{S_{q 2}} \\
\cos (\theta) & -\cos (\theta) & -\sin (\theta) & -\sin (\theta) \\
-\sin (\theta) & \sin (\theta) & -\cos (\theta) & -\cos (\theta) \\
Y_{1} & -\Upsilon_{2} & \left(d_{n 1}-l_{1}\right) & -\left(d_{n 2}-l_{2}\right) \\
1 & 0 & 0 & 0 \\
0 & 1 & 0 & 0 \\
0 & 0 & 1 & 0 \\
0 & 0 & 0 & 1
\end{array}\right]
\end{aligned}
$$

with $J_{S_{q i}}=J_{i}^{T} r_{Y}-r_{i} e_{i}$, for $i=1,2$. 
Taking the inner product between $\dot{z}$ and (3.8) yields

$$
\begin{gathered}
\frac{d}{d t}\left(K(z, \dot{z})+P(z)+P_{\mathrm{art}}(z)\right)=-\dot{z}^{T} F \dot{z}, \\
\frac{d}{d t} E_{T}(z, \dot{z})=-\sum_{i=1,2}\left(\dot{q}_{i}^{T} c_{i} \dot{q}_{i}\right),
\end{gathered}
$$

where $P_{\text {art }}(z)=\left(f_{d} / 2 \bar{l}\right) \Delta Y^{2}-\sum_{i} \int_{0}^{d_{n i}} f_{d} d(\xi)$ is called artificial potential energy. Then, the system satisfys the passivity condition in closed loop.

Although $\dot{E}_{T}(z, \dot{z})$ is negative definite along the solution trajectories of fingers-object system, $E(z, \dot{z})$ cannot play a role of a Lyapunov function for the closed-loop system. Because it is neither define positive in the 26-dimensional state space $(z, \dot{z})$ nor in 18-dimensional constrained manifold $M_{18}$ defined by

$$
M_{18}=\left\{(z, \dot{z}): C_{t i}=0, \dot{C}_{t i}=0, C_{n i}=0, \dot{C}_{n i}=0\right\}, \quad \text { for } i=1,2
$$

In order to define a constrained manifold where the trajectories of the system guarantees a stable grasping; a first step is to show the boundedness of solutions in the closed loop. Considering that $C_{n i}=0$ and $C_{t i}=0$ we have that $(d / d t) C_{n i}=\dot{z}^{T}(\partial / \partial z) C_{n i}=0$ and $(d / d t) C_{t i}=\dot{z}^{T}(\partial / \partial z) C_{t i}=0$, respectively. In fact, if the matrix $A=\left[(\partial / \partial z) C_{n 1},(\partial / \partial z) C_{n 2}\right.$, $\left.(\partial / \partial z) C_{t 1},(\partial / \partial z) C_{t 2}\right]$ from previous expressions we have that $0=A^{T} \dot{z}$. Then,

$$
0=\frac{d}{d t}\left(A^{T} \dot{z}\right)=A^{T} \ddot{z}+\dot{A}^{T} \dot{z} .
$$

Now, if we multiply (3.8) by $A^{T} H^{-1}$ we obtain that

$$
\Delta \lambda=\left(A^{T} H^{-1} A\right)^{-1}\left[-\dot{A}^{T} \dot{z}+A^{T} H^{-1}(C \dot{z}+P(z)-D \Delta Y+F \dot{z})\right],
$$

where $A^{T} \ddot{z}=-\dot{A}^{T} \dot{z}$ from (3.12). Taking into account that $\dot{z}, \Delta Y, d_{n i}, d_{t i}$, and $\phi_{i}$ are bounded due to $E_{T}(z, \dot{z}) \leq 0$; we have that $\Delta \mathcal{l}$ is bounded under assumption that the matrix $A$ in nondegenerate. Then, from (3.8) we have that $\ddot{z}$ is uniformly bounded which implies that $\dot{z}$ is uniformly continuous. Now, given that $\dot{q} \in L_{2}$ from (3.10) by Barbalat lemma [32] we have that $\dot{q} \rightarrow 0$ as $t \rightarrow 0$ which implies that $\dot{z} \rightarrow 0$ as $t \rightarrow 0$. Due to $\dot{z}$ and $\Delta Y$ are uniformly continuous, we have that $\ddot{z}$ is uniformly continuous too. This in turn implies that $\ddot{z} \rightarrow 0$ at $t \rightarrow \infty$ and (3.8) will be defined as

$$
P(z)=[A, D]\left[\begin{array}{l}
\Delta \lambda \\
\Delta Y
\end{array}\right]
$$

Assuming that the matrix $[A, D]$ is nondegenerate, a point that minimizes the right side of the (3.14) on $M_{18}$ exists. The unique critical point $\left(z^{*}\right)$ that minimizes (3.14) can be defined as

$$
\Delta \mathcal{\lambda}=0_{4 \times 1}, \quad \Delta Y=0 .
$$


This means that the closed-loop trajectories converge to critical point in the equilibrium point manifold (EP) defined as

$$
M_{4}=\left\{(z): C_{t i}=0, C_{n i}=0, \Delta Y \rightarrow 0, \Delta f_{i} \rightarrow 0, \Delta \lambda_{i} \rightarrow 0\right\}, \quad \text { for } i=1,2
$$

Hence, the total potential energy is minimizing and the object is held securely. It is important to notice that initial conditions of the system and the length of the object are very important parameters to guarantee the convergence on $M_{4}$. Furthermore, the matrix $[A, D]$ is nondegenerate if $J_{1}$ and $J_{2}$ are full rank matrices, that is, the matrix is degenerate if $q_{i 2}=q_{i 3}=0$ or $q_{i 2}=q_{i 3}=\pi$ for $i=1,2$. These joint values represent a special configuration of the fingers which can be excluded as a possible configuration in the manipulation tasks. At the same time, it is possible to show that the matrix $A$ is nondegenerate if the $J_{1}$ and $J_{2}$ are full rank matrices.

Now, we are in conditions to define the following.

(i) Let the neighborhood $N_{26}\left(z^{*}, r_{0}\right)$ singularity-free be with a radius $r_{0}>0$ around the critical point $\left(z^{*}, 0\right)$ defined as

$$
N_{26}\left(z^{*}, r_{0}\right)=\left\{(z, \dot{z}): \frac{1}{2} \Delta p^{T} H_{0} \Delta p+\sum_{i=1,2} \frac{1}{2} \Delta q_{i}^{T} H_{i}\left(q_{i}\right) \Delta q_{i}, \leq r_{0}^{2}\right\}
$$

where $\Delta p=p-p_{d}, \Delta q=q_{i}-q_{d}$. That is, singular configurations of the soft finger tips are avoided inside the neighborhood $N_{26}\left(z^{*}, r_{0}\right)$ provide that $r_{0}$ is chosen adequately.

(ii) Let the neighborhood $N_{18}\left(z^{*}, r_{0}\right)$ around the critical point be where the closed-loop trajectories remain with $\delta>0$, that is,

$$
N_{18}\left(z^{*}, r_{0}\right)=\left\{(z, \dot{z}): E_{T} \leq \delta,(z, \dot{z}) \in M_{18}\right\}
$$

(iii) Definition (see [26]) If for any given $\varepsilon>0$ there exists $\delta(\varepsilon)>0$ and another constant $r_{1}>0$ being less than $r_{0}$ and independent of $\varepsilon$ such that the solution tracking trajectories starting from any initial condition $(z(0), \dot{z}(0))$ lying on $N_{18}(\delta(\varepsilon)) \cap N_{26}\left(r_{1}\right)$ remains on $N_{18}(\varepsilon) \cap N_{26}\left(r_{0}\right)$ and converges asymptotically to the set $M_{4} \cap N_{18}(\varepsilon)$ as $t \rightarrow \infty$, then it is called that the state $\left(z^{*}, 0\right)$ is stable.

Finally, we have the following result.

Theorem 3.1. Considering that the desired reference state $\left(z^{*}, 0\right)$ and initial state $(z(0), \dot{z}(0))$ lying on $M_{4}$. The trajectories in the closed-loop system remains on $N_{18}(\varepsilon) \cap N_{26}\left(r_{0}\right)$ and converges asymptotically to the set $M_{4} \cap N_{26}(\varepsilon)$ as $t \rightarrow \infty$ under assumption that the matrix $\left[A, D_{\Theta}\right]$ is nondegenerate in a neighborhood $N_{26}\left(r_{0}\right)$. Thus, is assured that $\Delta f_{i} \rightarrow 0, Y_{1}-Y_{2} \rightarrow 0, \Delta \lambda_{i} \rightarrow 0$ as $t \rightarrow \infty$. 
Remark 3.2. Once the forces applied to the object have been compensated to hold it stably, the object can be rotated to the desired angle using the superposition principle, that is, the control law now is defined as

$$
u_{i}=u_{f c i}+u_{\theta c i}
$$

Premultiplying $\dot{q}_{i}^{T}$ by $u_{\theta c i}$ we have that

$$
\sum_{i=1}^{2} \dot{q}_{i}^{T} u_{\theta c i}=\frac{d}{d t}\left(E_{0}\right)
$$

where $E_{0}=(1 / 2) \beta(\overline{\Delta \tan (\theta)})^{2}$. This means that there exists a constant $\eta$ such that

$$
\int_{0}^{t}\left(\sum_{i=1}^{2} \dot{q}_{i}^{T} u_{\theta c i}\right) d \psi=E_{0}(t)-E_{0}(0) \geq \eta,
$$

where $\eta=-E_{0}(0)$.

Now, taking the inner product between $\dot{z}$ and the closed-loop system equations with $u_{i}$ defined in (3.19) we have that

$$
\frac{d}{d t} E_{T 1}=-\sum_{i=1,2}\left(\dot{q}_{i}^{T} c_{i} \dot{q}_{i}\right)
$$

where $E_{T 1}=K+P+P_{\text {art1 }}$ and $P_{\text {art1 }}=P_{\text {art }}+(1 / 2) \beta(\overline{\Delta \tan (\theta)})^{2}$. As in stable grasping, the closed-loop trajectories converge to critical point on a constrained manifold where $\Delta \lambda_{i} \rightarrow 0$, $\Delta f_{i} \rightarrow 0, \Delta Y \rightarrow 0$ and $\overline{\Delta \tan (\theta)} \rightarrow 0$ as $t \rightarrow \infty$.

Remark 3.3. Now, when the stable grasping and object orientation tasks has been established, the objective will be to move the object to desired coordinates $x_{d}$ by the following control law

$$
u_{x i}=-\frac{r_{x}}{2}\left(\bar{x}-x_{d}\right) \frac{\partial x_{i}}{\partial q_{i}}
$$

where $\gamma_{x}>0, x_{d}>0, x_{i}$ is the Cartesian coordinates and $\bar{x}$ is the estimated position of the object which is defined as an average distance between center positions of the deformable fingertips, that is,

$$
\bar{x}=\frac{x_{1}+x_{2}}{2} .
$$

Using the superposition principle, the control law for the stable grasping and object manipulation is defined as

$$
u_{i}=u_{f c i}+u_{\theta c i}+u_{x i} \quad \text { for } i=1,2
$$


As in the previous case, if we multiplying $u_{x i}$ by $\dot{q}^{T}$ we have that

$$
\sum_{i=1}^{2} \dot{q}_{i}^{T} u_{x i}=\frac{d}{d t}\left(E_{1}\right)
$$

where $E_{1}=(1 / 2) \gamma_{x}\left(\bar{x}-x_{d}\right)^{2}$ is the shifting energy to move the object in $x$-coordinates. Consequently, exists a constant $\eta_{1}$ such that

$$
\int_{0}^{t}\left(\sum_{i=1}^{2} \dot{q}_{i}^{T} u_{x i}\right) d \psi=E_{1}(t)-E_{1}(0) \geq \eta_{1}
$$

where $\eta_{1}=-E_{1}(0)$.

Now, taking the inner product between $\dot{z}$ and the closed-loop system equations with $u_{i}$ defined in (3.25) we have

$$
\frac{d}{d t} E_{T 2}=-\sum_{i=1,2}\left(\dot{q}_{i}^{T} c_{i} \dot{q}_{i}\right)
$$

where $E_{T 2}=K+P+P_{\text {art2 }}$ and $P_{\text {art2 }}=P_{\text {art1 }}+(1 / 2) \gamma_{x}\left(\bar{x}-x_{d}\right)^{2}$. Then, the passivity condition is satisfy in the closed-loop. Hence, the closed-loop system trajectories converge to critical point on a constrained manifold where $\Delta \lambda_{i} \rightarrow 0, \Delta f_{i} \rightarrow 0, \Delta Y \rightarrow 0, \overline{\Delta \tan (\theta)} \rightarrow 0$, and $\bar{x} \rightarrow x_{d}$ as $t \rightarrow \infty$.

\section{Simulation Results}

In order to demonstrate usefulness of our scheme for stable grasping and object orientation, numerical simulations were carried out on a pair of deformable fingertips in the horizontal plane, see Figure 1. The simulations were implemented on stiff numerical solver on Matlab R2007b, under $1 \mathrm{~ms}$ sampling time. Additionally, to approximate the holonomic constraints was used the Constrained Stabilization Method (CSM) [33].

The physical parameters of the fingers and object are shown in Table 1 where $l_{i j}, m_{i j}$ and $I_{i j}$ are the length, mass and moment of inertia of the link $j=1,2,3$ for the finger $i=1,2$, and $M, I, l$ are the mass, moment of inertia and the length of the object, respectively (Table 2). Moreover, $L=0.64[\mathrm{~m}]$ is the distance between fingers, $E=50000\left[\mathrm{~N} / \mathrm{m}^{2}\right]$ is the Young's modulus of the fingertips and $r_{i}=0.01[\mathrm{~m}]$ is the radius of the hemispherical finger tip for $i=1,2$.

The simulation study is divided in three steps. As first step before any manipulation task is necessary to guarantee that the stable grasp is achieved through the control law defined as $u_{i}=u_{f c i}$. The initial conditions used in the simulations are $q_{1}(0)=(30,91.61$, $71.24)^{T}, q_{2}(0)=(30,91.61,71.24)^{T},(x(0), y(0))=(0.032,0.047)[\mathrm{m}], \theta(0)=0$, and $\left(d_{n 1}, d_{n 2}\right.$, $\left.d_{t 1}, d_{t 2}\right)=(0.0025,0.0025,0,0)[\mathrm{m}]$ which establish the following conditions: $f_{1}(0)=1.1[\mathrm{~N}]$, $f_{2}(0)=1.1[\mathrm{~N}] \Delta Y(0)=0[\mathrm{~m}]$. For reference, these initial conditions are called normal initial conditions $\left(\mathrm{CI}_{N}\right)$. 
Table 1: Physical parameters.

\begin{tabular}{lc}
\hline Parameter & Value \\
\hline$l_{11}=l_{21}$ & $0.05(\mathrm{~m})$ \\
$l_{12}=l_{22}$ & $0.04(\mathrm{~m})$ \\
$l_{13}=l_{23}$ & $0.03(\mathrm{~m})$ \\
$m_{11}=m_{21}$ & $0.05(\mathrm{~kg})$ \\
$m_{12}=m_{22}$ & $0.03(\mathrm{~kg})$ \\
$m_{13}=m_{23}$ & $0.02(\mathrm{~kg})$ \\
$I_{11}=I_{21}$ & $1.4167 \times 10^{-5}\left(\mathrm{~kg} \mathrm{~m}^{2}\right)$ \\
$I_{12}=I_{22}$ & $6.25 \times 10^{-6}\left(\mathrm{~kg} \mathrm{~m}^{2}\right)$ \\
$I_{13}=I_{23}$ & $3 \times 10^{-6}\left(\mathrm{~kg} \mathrm{~m}^{2}\right)$ \\
\hline
\end{tabular}

Table 2: Parameters of the object.

\begin{tabular}{lc}
\hline Parameter & Value \\
\hline$M$ & $0.02(\mathrm{~kg})$ \\
$I$ & $5.67 \times 10^{-6}\left(\mathrm{~kg} \mathrm{~m}^{2}\right)$ \\
$l$ & $0.03(\mathrm{~m})$ \\
\hline
\end{tabular}

In Figure 3 we observe that the forces on the object $\Delta f_{i}$ and $\Delta \lambda_{i}$ converge rapidly to the desired values using the normal initial conditions defined previously. At the same time, the fast convergence of $\Delta Y$ to zero is shown in Figure 3. On the other hand, the Figures 4, 5, and 6 show the performance of $\Delta f_{i}, \Delta \lambda_{i}$, and $\Delta Y$ for different values of damping gains $c_{i}$. Notice that the damping gains are related with the convergence velocity towards equilibrium point that minimizes the potential energy of the system. However, the system takes longer to converge and several oscillations arise when the damping gains are small. The control parameters used in this step are $f_{d}=1[\mathrm{~N}]$ and $c_{1}=c_{2}=0.01[\mathrm{Nms} / \mathrm{rad}]$.

Finally, the Figures 7,8 , and 9 show the convergence of $\Delta f_{i}$ and $\Delta Y$ to zero under more extreme initial conditions. The initial conditions used in these simulations are $\mathrm{CI}_{1}$ and $\mathrm{CI}_{2}$ which are defined as $q_{1}(0)=(30,95.65,64.93)^{T}, q_{2}(0)=(30,100.45,76.09)^{T}, \Delta Y(0)=$ $0.01[\mathrm{~m}]$, and $q_{1}(0)=(30,93.73,68.32)^{T}, q_{2}(0)=(30,102.16,72.78)^{T},\left(d_{n 1}, d_{n 2}, d_{t 1}, d_{t 2}\right)=$ $(0.0013,0.0037,0,0)[\mathrm{m}], \Delta Y(0)=0.01[\mathrm{~m}]$, respectively.

Notice that the convergence to zero of $\Delta f_{i}$ and $\Delta Y$ present a transient responses for different initial conditions, but still converge to the desired values in few seconds. Additionally, the control objectives converge in different times. Specially, the convergence of $\Delta Y$ takes more time for establishing a stable grasping when the initial conditions are more extreme, $\mathrm{CI}_{2}$. Thus, it is possible to describe the stable grasping in two phases. In the first phase, the stabilization of the normal and tangential forces on object is performed while the stabilization of the rotational moments to stop the angular motion of the object is carried out in a second phase.

Once the all forces applied to the object have been compensated, it is possible to execute any manipulation task. In this case the rotation of the object, to a desired angle $\theta_{d}$, will be realized. Using the superposition principle the control law is given as $u_{i}=u_{f c i}+u_{\theta c i}$. The convergence to zero of $\Delta f_{i}, \Delta \lambda_{i}, \Delta Y$, and $\Delta \theta$ using the $\mathrm{CI}_{N}$ initial conditions are shown in Figures 10 and 11. It is important to notice the special role of $Y_{1}-Y_{2}$ as a parameter to increase the dexterity. In this case, the convergence of $\Delta Y$ is closely associated to object orientation 

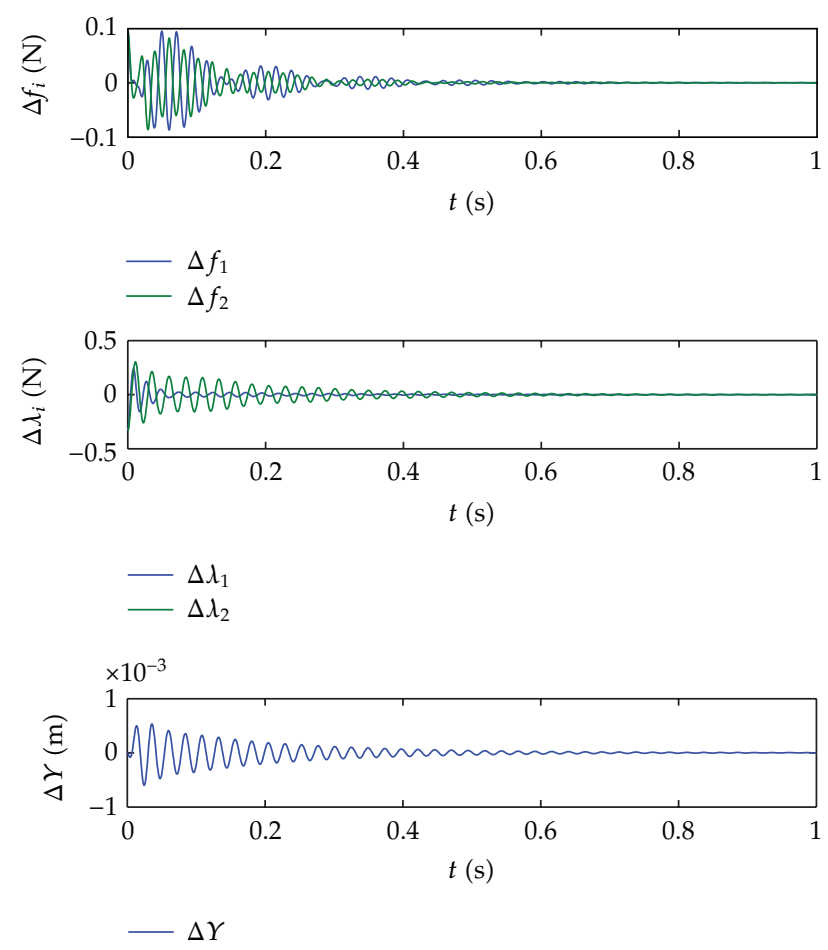

Figure 3: Convergence of $\Delta f_{i}, \Delta \lambda_{i}$, and $\Delta Y$ for $i=1,2$.

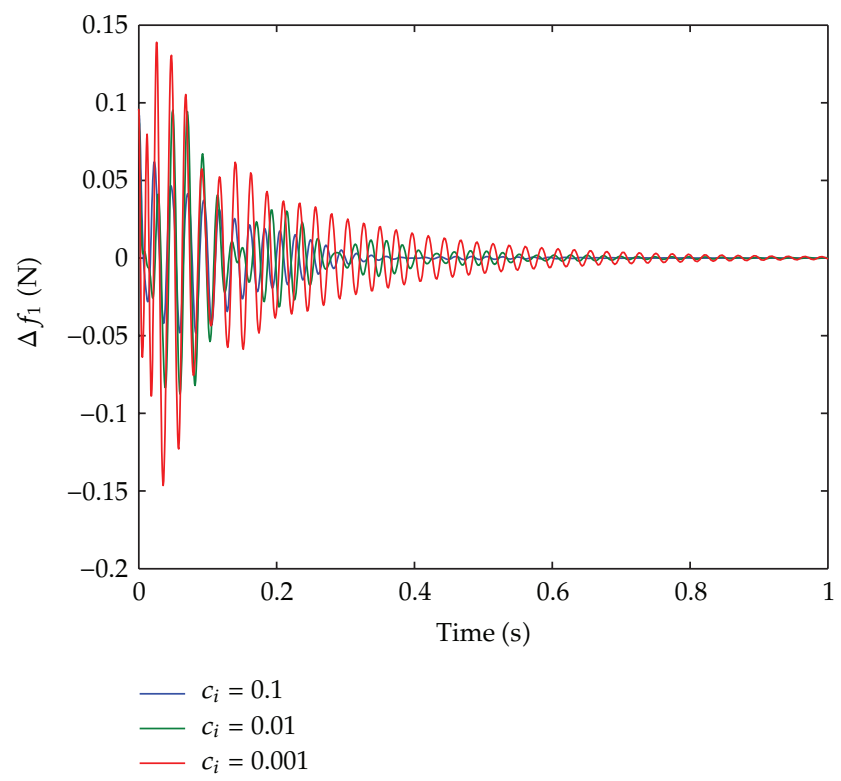

Figure 4: Convergence of $\Delta f_{i}$ considering several values of $c_{i}$. 


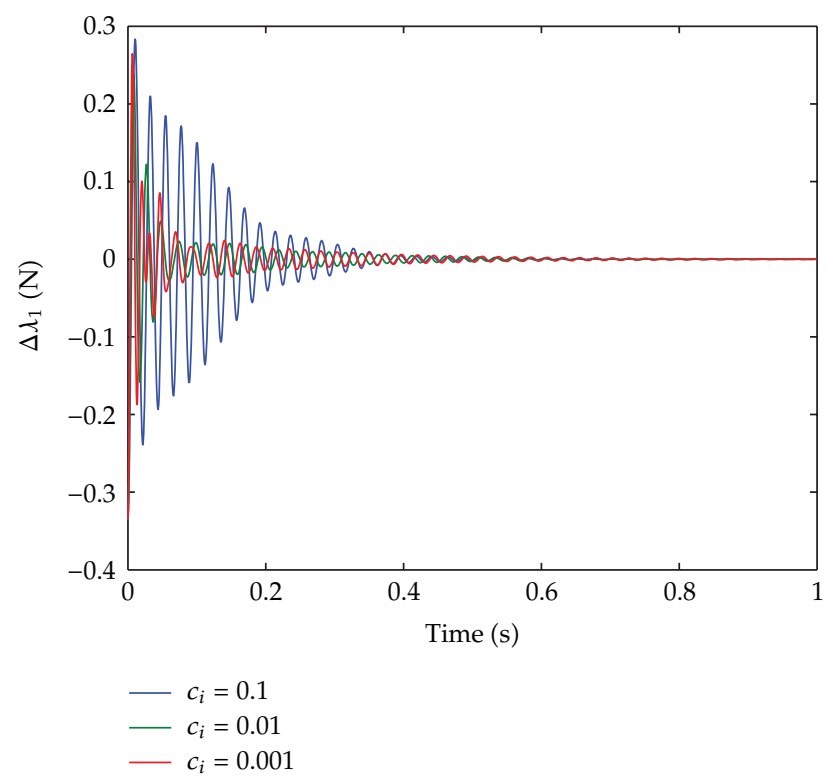

Figure 5: Convergence of $\Delta \lambda_{i}$ considering several values of $c_{i}$.

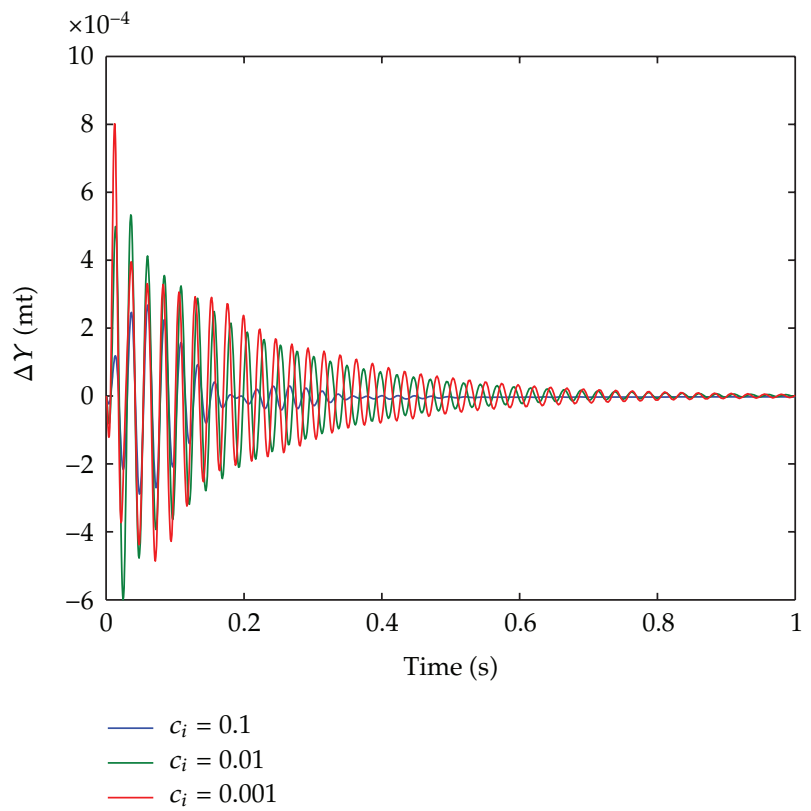

Figure 6: Convergence of $\Delta Y$ considering several values of $c_{i}$.

through $\overline{\tan (\theta)}$. The control parameters using in this second step are $f_{d}=1[\mathrm{~N}], c_{1}=c_{2}=$ $0.01[\mathrm{Nms} / \mathrm{rad}], \beta=0.1[\mathrm{~N} / \mathrm{rad}]$, and $\theta_{d}=-10(\pi / 180)[\mathrm{rad}]$.

As the final step of this study, a stable grasping and object manipulation which include the orientation and translation of the object to a desired reference is presented. Using a superposition principle, the control law for this step is defined as $u=u_{f c i}+u_{\theta c i}+u_{\text {tras }}$. 


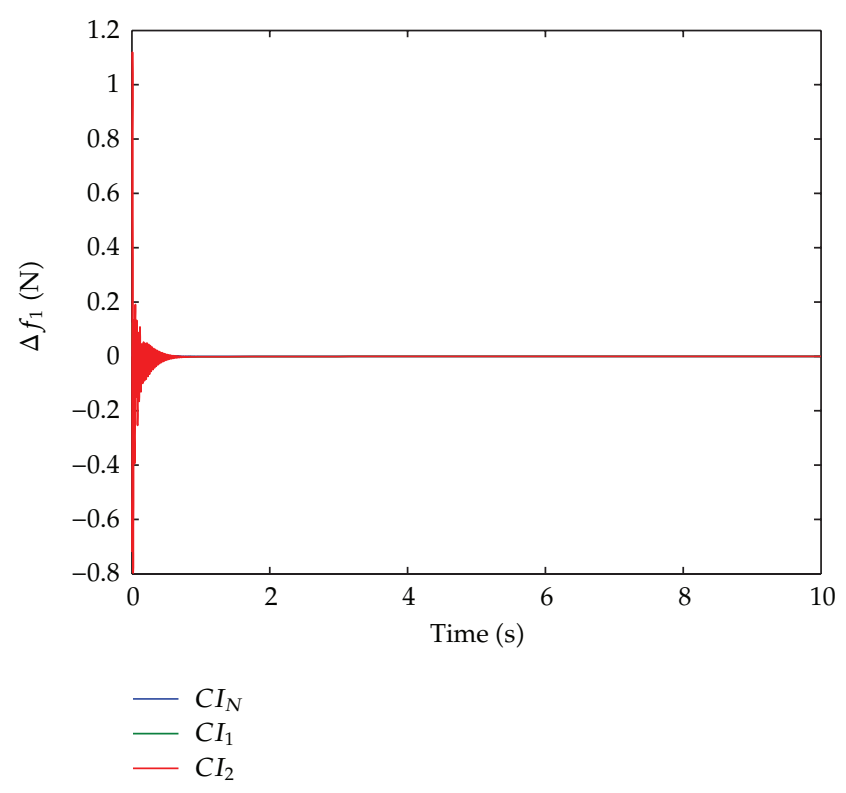

Figure 7: Convergence of $\Delta f_{i}$ considering different initial conditions.

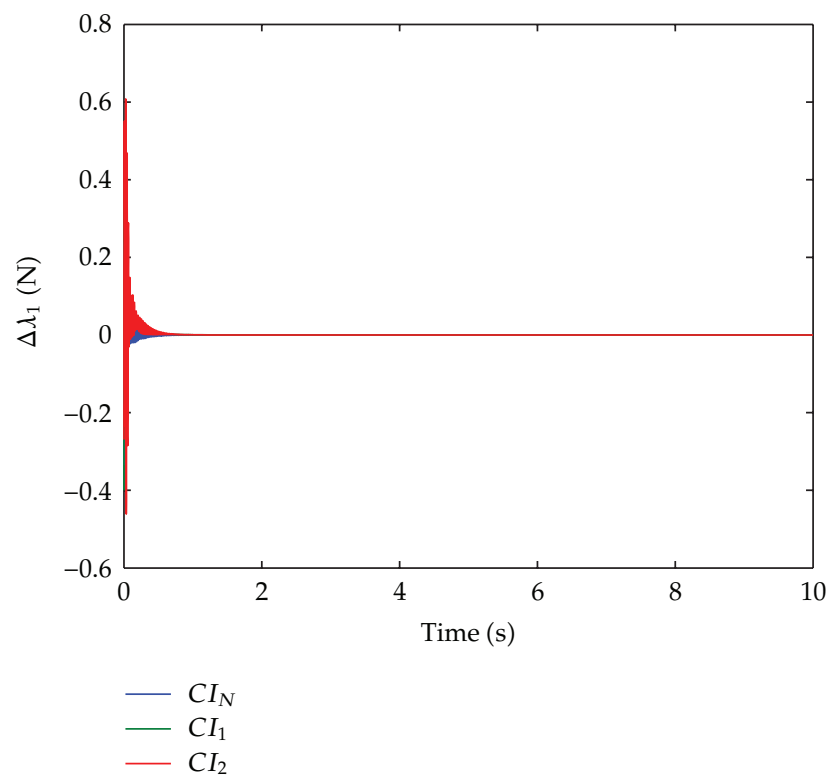

Figure 8: Convergence of $\Delta \ell_{i}$ considering different initial conditions.

The Figures 12, 13, and 14 show the convergence of $\Delta f_{i}, \Delta \ell_{i}, \Delta Y$, and $\Delta \theta$ to zero using $\mathrm{CI}_{N}$ initial conditions. The control parameters used in this step are $f_{d}=1[\mathrm{~N}], c_{1}=$ $c_{2}=0.01[\mathrm{Nms} / \mathrm{rad}], \beta=0.1[\mathrm{~N} / \mathrm{rad}], \theta_{d}=-10(\pi / 180)[\mathrm{rad}], \gamma_{x}=50[\mathrm{~N} / \mathrm{m}]$, and $\bar{x}_{d}=$ $\bar{x}_{0}+0.01[\mathrm{~m}]$. 


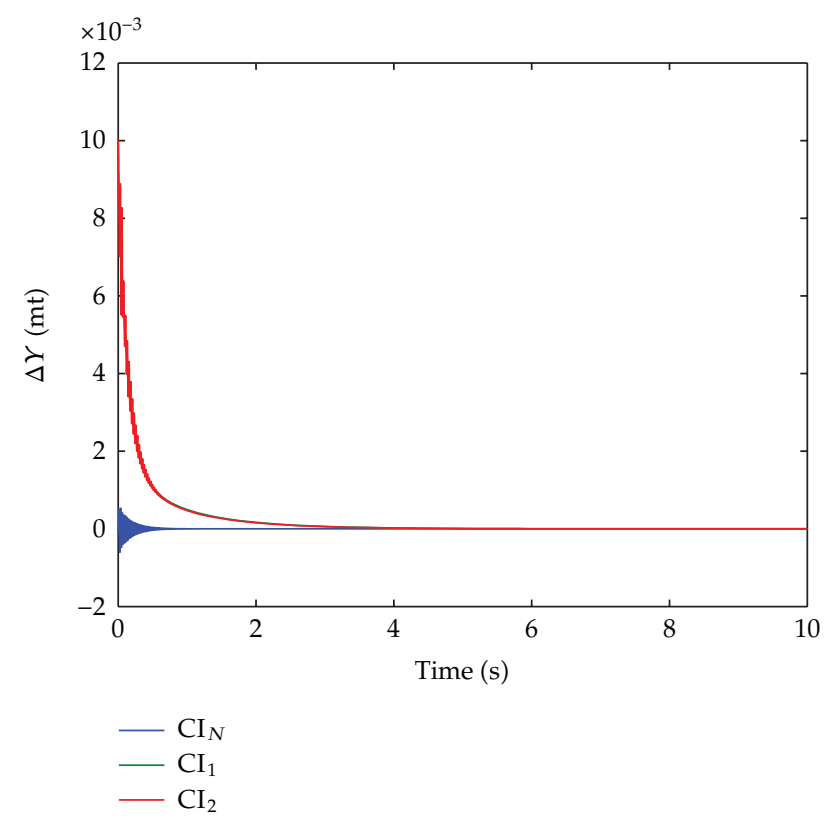

Figure 9: Convergence of $\Delta Y$ considering different initial conditions.
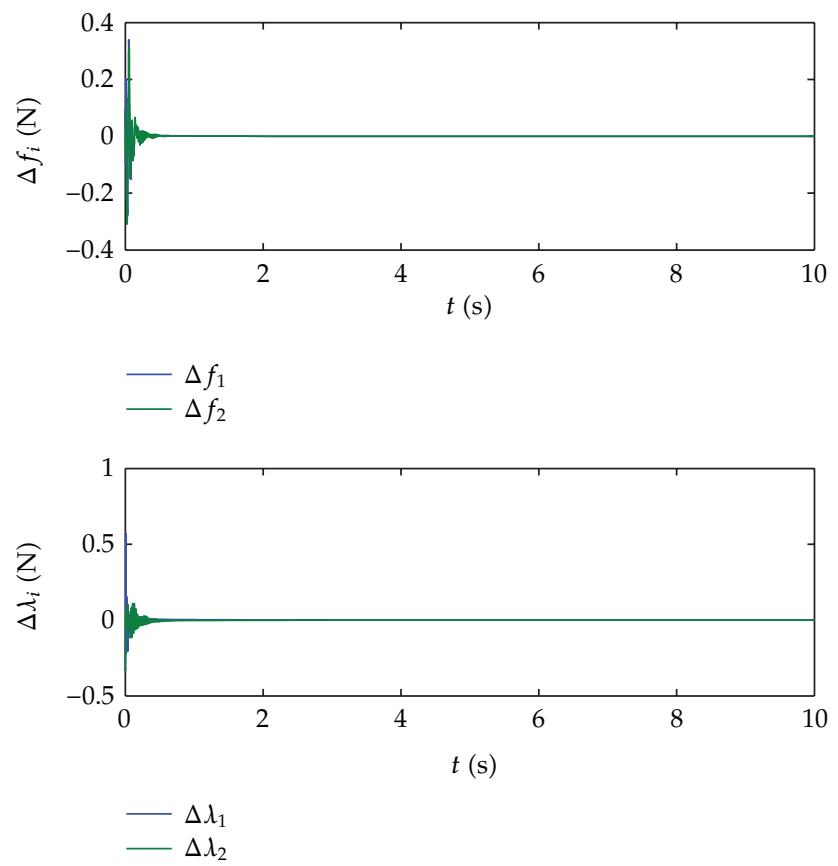

Figure 10: Convergence of $\Delta f_{i}$ and $\Delta \lambda_{i}$ for $i=1,2$. 


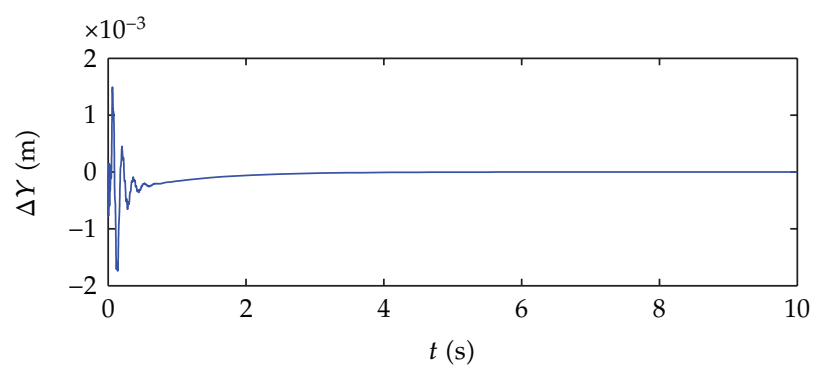

$-\Delta Y$

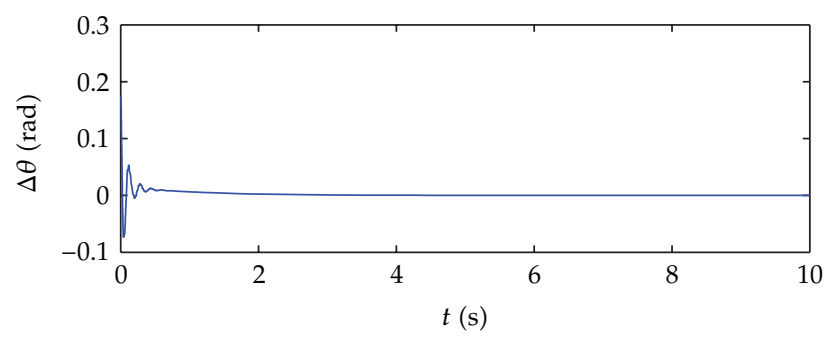

$-\Delta \theta$

Figure 11: Convergence of $\Delta Y$ and $\Delta \theta$ for $i=1,2$.
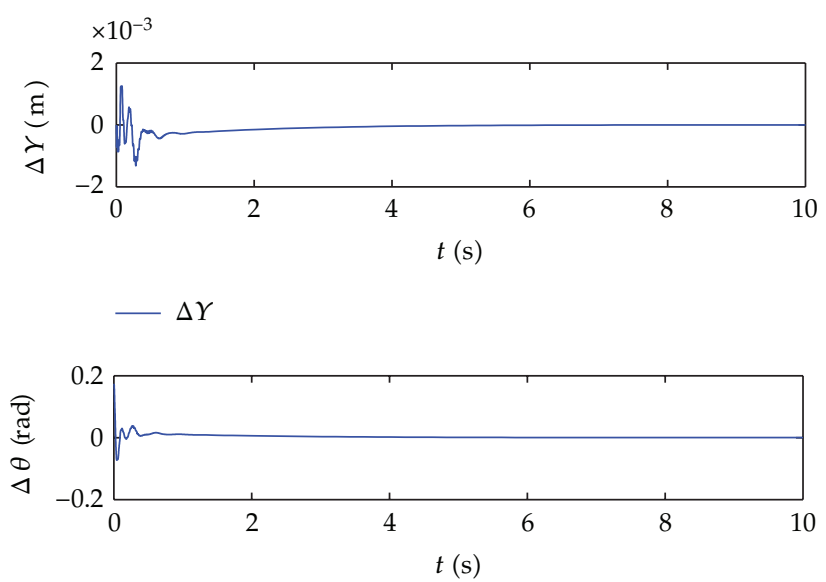

$-\Delta \theta$

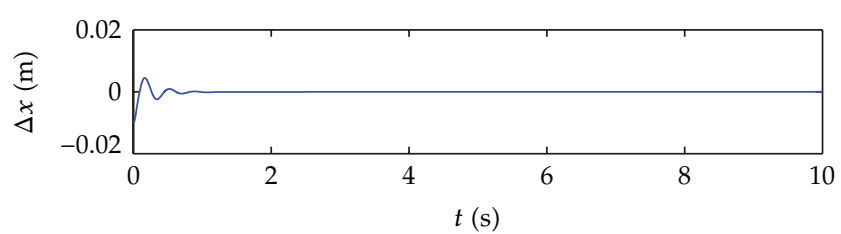

$-\Delta x$

Figure 12: Convergence of $\Delta Y, \Delta \theta$, and $\Delta x$ for $i=1,2$. 

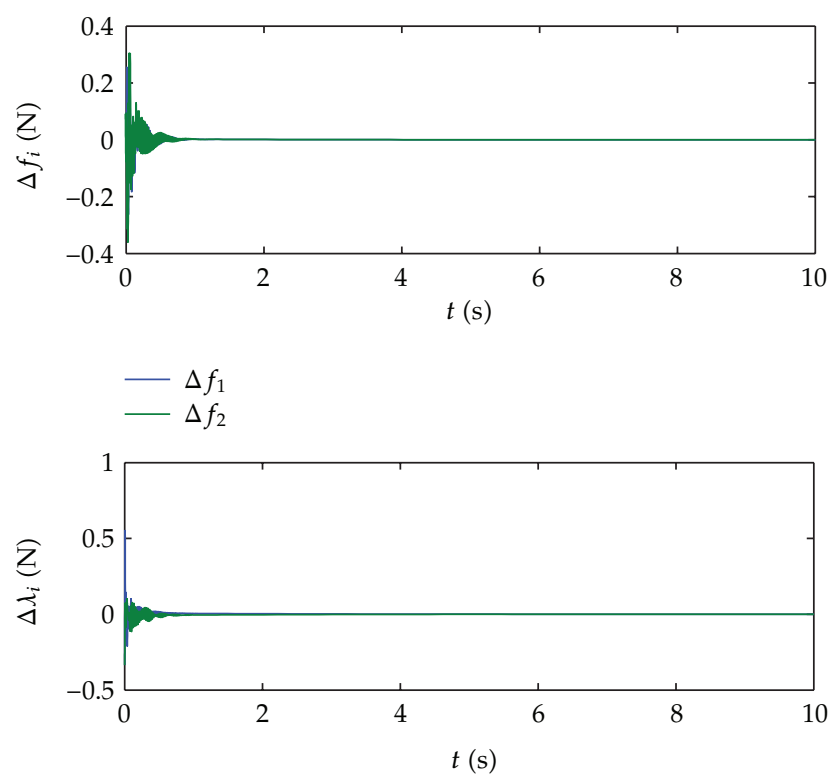

$-\Delta \lambda_{1}$

$-\Delta \lambda_{2}$

Figure 13: Convergence of $\Delta f_{i}$ and $\Delta \lambda_{i}$ for $i=1,2$.

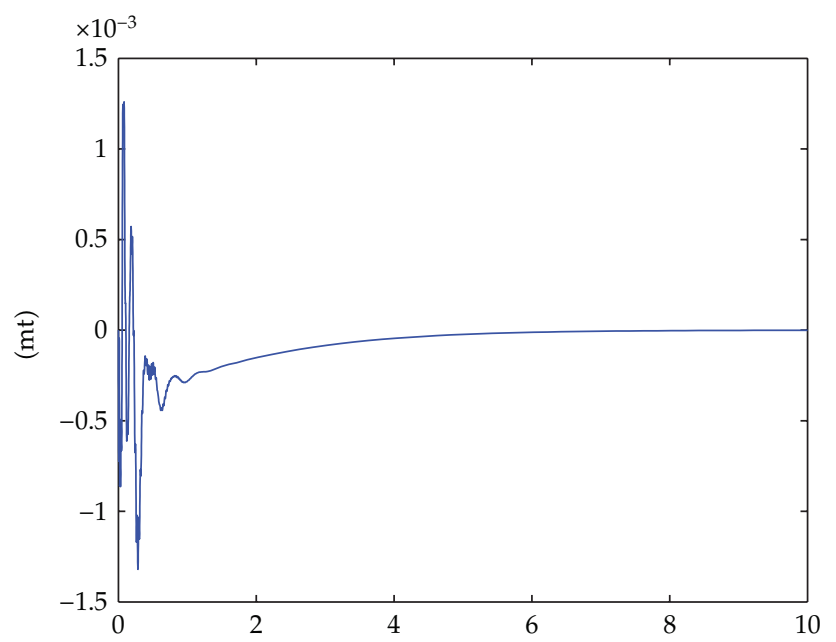

(s)

$-\Delta Y$

Figure 14: Convergence of $\Delta Y$ for $i=1,2$.

\section{Conclusions}

A scheme to grasp and manipulate an object using soft tip fingers is presented. In order to include more characteristics of the human fingertips a parallel deformation model is used. The control law proposed ensures stability on a constrained manifold. Furthermore, 
the control law avoids information of the radius of the tips and the convergence to the desired force value is guaranteed.

Numerical simulations, on a pair of deformable fingertips in horizontal plane, allow us to visualize the convergence of the closed-loop trajectories to the desired point. In addition, the special role of $\Delta Y$ in the manipulation task and the effects of the superposition principle that have been observed.

\section{Acknowledgments}

This work was partially supported by CONICYT, Departamento de Relaciones Internacionales, Programa de Cooperación Científica Internacional, CONICYT/CONACYT 2011380, and the Universidad de los Andes, Chile, FAI project ICI-002-11.

\section{References}

[1] J. R. Flanagan and R. S. Johansson, Hand Movements, Encyclopedia of the Human Brain, vol. 2, Academic Press, San Diego, Calif, USA, 2002.

[2] A. M. Okamura, N. Smaby, and M. R. Cutkosky, "Overview of dexterous manipulation," in Proceedings of the IEEE International Conference on Robotics and Automation (ICRA '00), pp. 255-262, April 2000.

[3] J. K. Salisbury and B. Roth, "Kinematic and force analysis of articulated mechanical hands," Journal of Mechanisms, Transmissions, and Automation in Design, vol. 105, no. 1, pp. 35-41, 1983.

[4] E. A. Al-Gallaf, "Multi-fingered robot hand optimal task force distribution: neural inverse kinematics approach," Robotics and Autonomous Systems, vol. 54, no. 1, pp. 34-51, 2006.

[5] A. Bicchi, "Hands for dexterous manipulation and robust grasping: a difficult road toward simplicity," IEEE Transactions on Robotics and Automation, vol. 16, no. 6, pp. 652-662, 2000.

[6] W. Gueaieb, S. Al-Sharhan, and M. Bolic, "Robust computationally efficient control of cooperative closed-chain manipulators with uncertain dynamics," Automatica, vol. 43, no. 5, pp. 842-851, 2007.

[7] J.-W. Li, H. Liu, and H.-G. Cai, "On computing three-finger force-closure grasps of 2-D and 3-D objects," IEEE Transactions on Robotics and Automation, vol. 19, no. 1, pp. 155-161, 2003.

[8] H. Kawasaki, R. Bin Ramli, and S. Ueki, "Decentralized adaptive coordinated control of multiple robot arms for constrained tasks," Journal of Robotics and Mechatronics, vol. 18, no. 5, pp. 580-588, 2006.

[9] T. Naniwa and K. Wada, "Experimental study of coordinated control of multifingered hands with the kinetostatic filtering method," Advanced Robotics, vol. 19, no. 2, pp. 191-206, 2005.

[10] E. Rimon, "A curvature-based bound on the number of frictionless fingers required to immobilize three-dimensional objects," IEEE Transactions on Robotics and Automation, vol. 17, no. 5, pp. 679-697, 2001.

[11] X.-Z. Zheng, R. Nakashima, and T. Yoshikawa, “On dynamic control of finger sliding and object motion in manipulation with multifingered hands," IEEE Transactions on Robotics and Automation, vol. 16, no. 5, pp. 469-481, 2000.

[12] A. B. A. Cole, J. E. Hauser, and S. S. Sastry, "Kinematics and control of multifingered hands with rolling contact," Institute of Electrical and Electronics Engineers, vol. 34, no. 4, pp. 398-404, 1989.

[13] A. Nakashima, K. Nagase, and Y. Hayakawa, "Control of contact points of two- fingered robot hand with manipulating an object," in Proceedings of the ICASE/SICE Joint Workshop, pp. 120-125, 2004.

[14] A. I. Tuneski and G. Rafajlovski, "Adaptive control of multiple robots manipulation on dynamical environment," in Proceedings of the 7th IEEE International Conference on Emerging Technologies and Factory Automation (ETFA '99), vol. 1, pp. 623-632, October 1999.

[15] M. Zribi, J. Chen, and M. S. Mahmoud, "Coordination and control of multi-fingered robot hands with rolling and sliding contacts," Journal of Intelligent and Robotic Systems, vol. 24, no. 2, pp. 125-149, 1999.

[16] C. Borst, M. Fischer, and G. Hirzinger, "Grasp planning: how to choose a suitable task wrench space," in Proceedings IEEE International Conference on Robotics and Automation, pp. 319-325, May 2004.

[17] I. Kao and F. Yang, "Stiffness and contact mechanics for soft fingers in grasping and manipulation," IEEE Transactions on Robotics and Automation, vol. 20, no. 1, pp. 132-135, 2004. 
[18] S. K. Song, J. B. Park, and Y. H. Choi, “Grasping control of 3-joint dual finger robot: lyapunov stability approach," in Proceedings of the American Control Conference (ACC '09), pp. 2879-2884, St. Louis, Mo, USA, June 2009.

[19] Y. Xue and I. Kao, "Dextrous sliding manipulating using soft fingertips," in Proceedings of the IEEE International Conference on Robotics and Automation, vol. 4, pp. 3397-3402, May 1994.

[20] P. Akella and M. Cutkosky, "Manipulating with soft fingers," in Proceedings of the IEEE International Conference on Robotics and Automation, pp. 767-769, 1989.

[21] K. B. Shimoga and A. A. Goldenberg, "Soft materials for robotic fingers," in Proceedings of the IEEE International Conference on Robotics and Automation, pp. 1300-1305, May 1992.

[22] I. Kao and F. Yang, "Stiffness and contact mechanics for soft fingers in grasping andmanipulation," IEEE Transactions on Robotics and Automation, vol. 20, no. 1, pp. 132-135, 2004.

[23] D. J. Montana, "Kinematics of contact and grasp," International Journal of Robotics Research, vol. 7, no. 3, pp. 17-32, 1988.

[24] Y. Yokokohji, M. Sakamoto, and T. Yoshikawa, "Vision-aided object manipulation by a multifingered hand with soft fingertips," in Proceedings of the IEEE International Conference on Robotics and Automation (ICRA '99), vol. 4, pp. 3201-3208, May 1999.

[25] T. Inoue and S. Hirai, Mechanics and Control of Soft-Fingered Manipulation, Springer, 2009.

[26] S. Arimoto, "Intelligent control of multi-fingered hands," Annual Reviews in Control, vol. 28, no. 1, pp. 75-85, 2004.

[27] M. Yoshida, S. Arimoto, and J. H. Bae, "Blind grasp and manipulation of a rigid object by a pair of robot fingers with soft tips," in Proceedings of the IEEE International Conference on Robotics and Automation (ICRA '07), pp. 4707-4714, Rome, Italy, April 2007.

[28] S. Arimoto, P. T. A. Nguyen, H. Y. Han, and Z. Doulgeri, "Dynamics and control of a set of dual fingers with soft tips," Robotica, vol. 18, no. 1, pp. 71-80, 2000.

[29] M. L. Latash and M. L. Zatsiorsky, "Principle of superposition in human prehension," in Advances in Robot Control, pp. 249-261, Springer, 2006.

[30] S. Arimoto, "Reduction of complexity in learning dexterous multi-fingered motions: a theoretical exploration into a future problem C. E. Shannon raised," Communications in Information and Systems, vol. 1, no. 1, pp. 1-14, 2001.

[31] H. Goldstein, C. P. Poole, and J. L. Safko, Classical Mechanics, Addison Wesley, 3rd edition, 2002.

[32] F. L. Lewis and C. T. Abdallaah, Control of Robot Manipulators, Macmillan, New York, NY, USA, 1994.

[33] J. Baumgarte, "Stabilization of constraints and integrals of motion in dynamical systems," Computer Methods in Applied Mechanics and Engineering, vol. 1, pp. 1-16, 1972. 


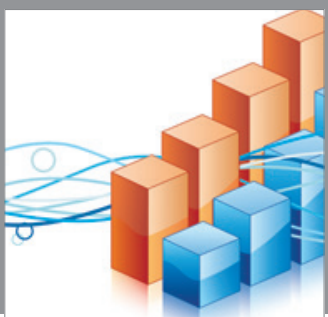

Advances in

Operations Research

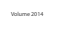

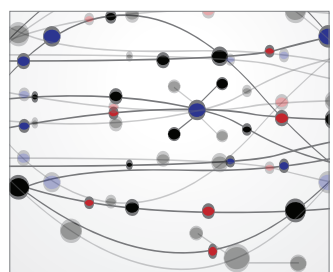

\section{The Scientific} World Journal
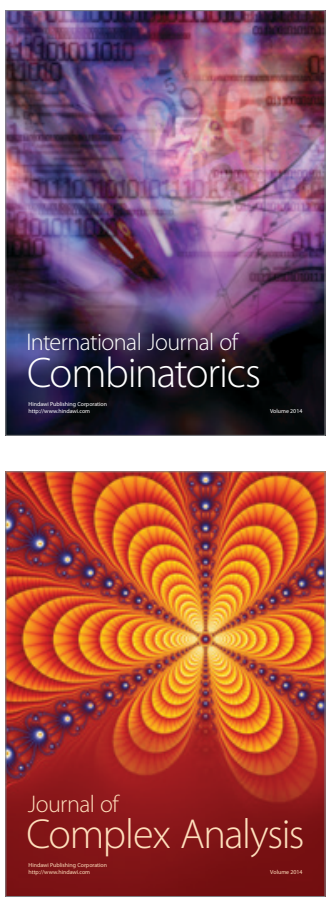

International Journal of

Mathematics and

Mathematical

Sciences
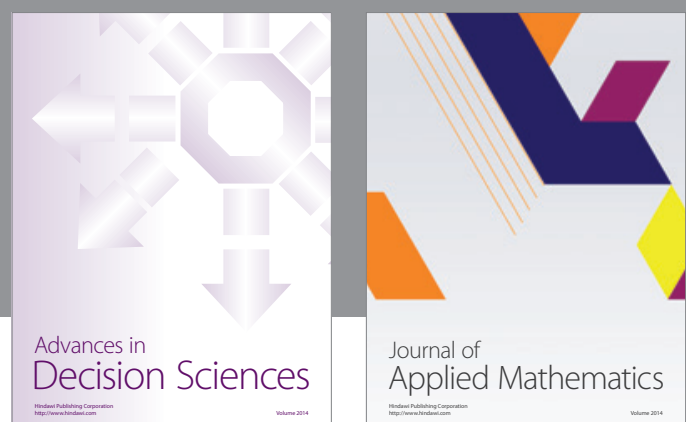

Journal of

Applied Mathematics
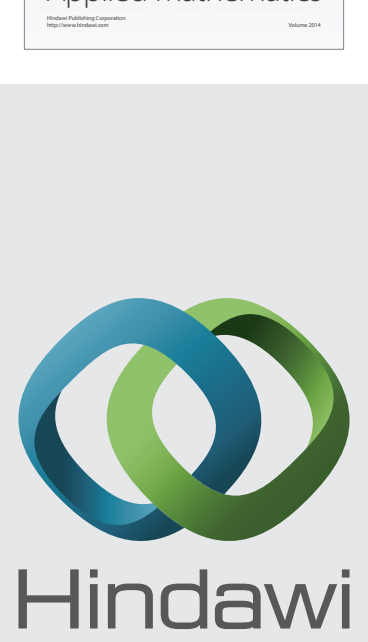

Submit your manuscripts at http://www.hindawi.com
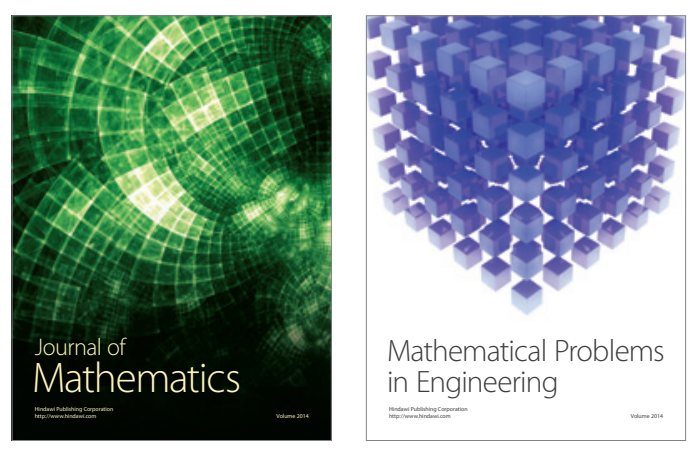

Mathematical Problems in Engineering
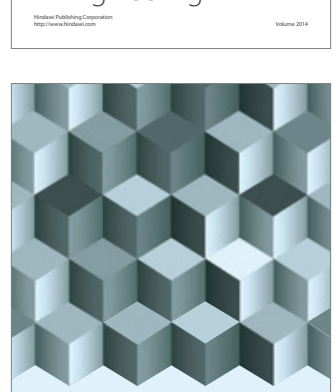

Journal of

Function Spaces
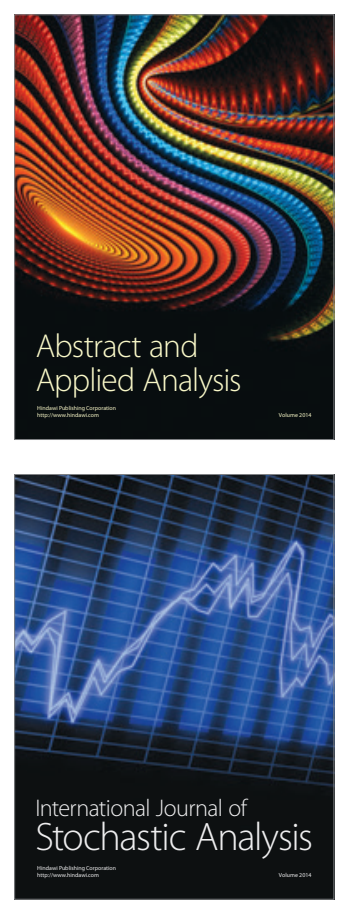

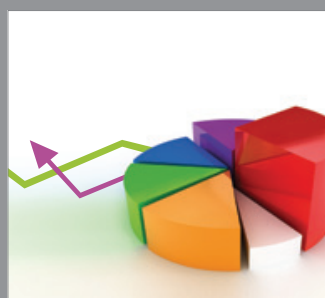

ournal of

Probability and Statistics

Promensencen
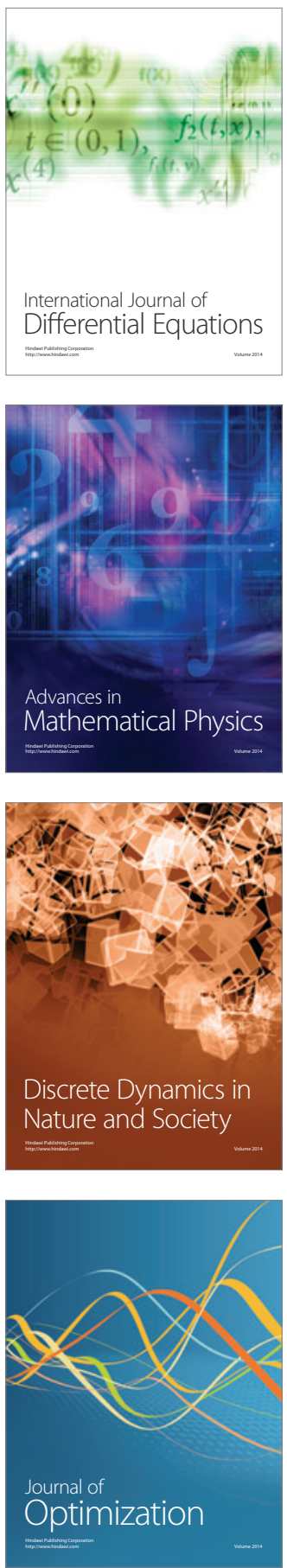